\title{
Basic Mechanisms of Antibiotic Resistance: Molecular Properties of Multidrug Transporters
}

\author{
Hendrik W. van Veen*, M.Sc., Ph.D., Monique Putman, M.Sc., \\ Wim van Klompenburg, Ph.D., Rene Heijne, M.Sc., Abelardo Margolles, Ph.D., \\ and Wil N. Konings, Ph.D.
}

\section{INTRODUCTION}

The selection of drug-resistant pathogenic microorganisms in hospitalized patients with serious infections such as pneumonia, urinary tract infections, skin infections, and bacteremia has generally been ascribed to the widespread use of antimicrobial agents $(1,2)$. Likewise, nutritive and therapeutic treatment of farm animals with antibiotics, amounting to half of the world's antibiotic output, has selected for drug resistant microorganisms that contaminate the food produced (3). Issues of concern regarding gram-negative bacteria include the extended drug resistance spectrum of $\beta$-lactamase-producing Escherichia coli, Salmonella typhimurium and Klebsiella pneumonia. Current concerns with gram-positive pathogens are increasing multidrug resistance in Listeria monocytogenes, methicillin-resistant Stalphylococcus aureus and penicillin-resistant Streptococcus pneumonia (for review, see J. Kellner and D.E. Low in this issue of the MJM). Besides bacteria, parasitic protozoa are also responsible for some of the most devastating and prevalent diseases of humans and domestic animals, such as malaria (Plasmodium spp.), (muco)cutaneous and visceral leishmaniasis (Leishmania spp.), African sleeping sickness (Trypanosoma brucei gambiense, Trypanosoma brucei rhodensiense), South-American Chagas' disease (Trypanosoma cruzi), amoebic dysentery (Entamoeba spp.), and toxoplasmosis (Toxoplasma spp.) (for review see (4)). Chemotherapeutic treatment of infections by these parasites is being eroded by multidrug resistance.

* To whom correspondence should be addressed: Groningen Biomolecular Sciences and Biotechnology Institute, Department of Microbiology, University of Groningen, Kerklaan 30, NL-9751 NN Haren, Netherlands. Fax: +31-50-3632158
With few new drugs in the pipeline, prevention and circumvention of microbial multidrug resistance are medical and veterinary priorities, which require insight into the molecular basis of microbial multidrug resistance.

The presence of multidrug resistant pathogenic and non-pathogenic microorganisms suggests that multidrug resistance did not arise recently in pathogens in response to antimicrobial chemotherapy. Toxic compounds have always been part of the natural environment in which microorganisms dwell. The development of strategies for life in this habitat has been crucial for survival of the cell. As a result, microorganisms can use various mechanisms to resist cytotoxic drugs. Microorganisms can eliminate the drug target in the cell through the alteration or replacement of molecules that are normally bound by the antibiotic (5). Alternatively, microorganisms can reduce the intracellular concentration of drugs by: (i) synthesizing enzymes that degrade antibiotics or that chemically modify, and so inactivate, the drugs (6), (ii) eliminating entry ports for hydrophilic drugs, such as outer membrane porins in gram-negative bacteria (7), and (iii) manufacturing drug efflux systems that export lipophilic drugs before these compounds have the chance to find their cellular targets $(4,8,9)$. Some of these drug efflux systems are fairly specific for a given drug or class of drugs, but the so-called multidrug transporters have specificity for compounds with very different chemical structures and cellular targets. Multidrug transporters can be amplified in drug resistant pathogenic microorganisms, and can shift their drug profiles, making them a menace to drug treatment.

Multidrug transporters are also found in mammals, in which they are a cause of multidrug resistance of tumor 


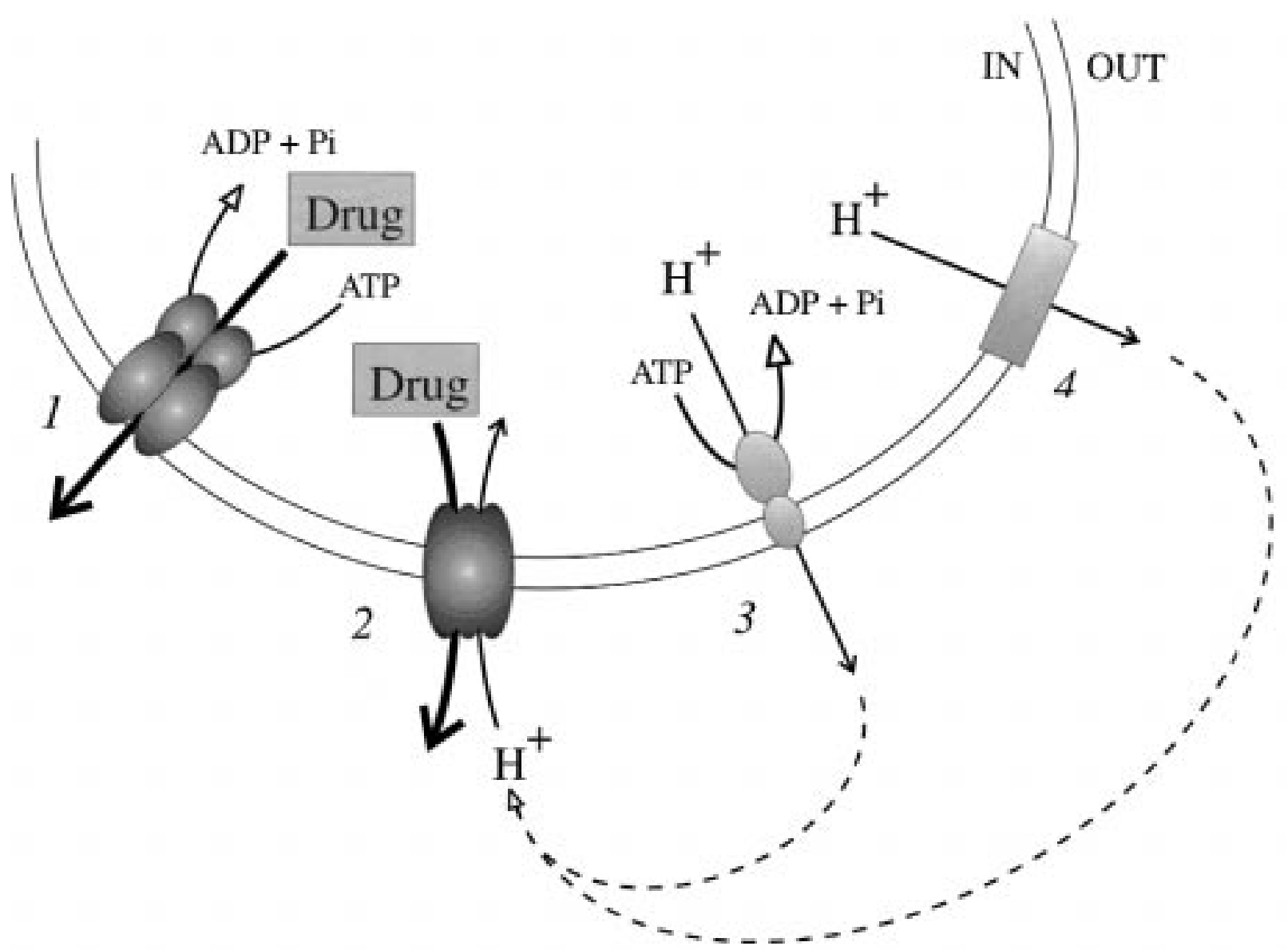

Figure 1. Schematic presentation of the two major classes of multidrug transporters in bacteria. (1) ABC-type multidrug transporters mediate the extrusion of drugs from the cell coupled to the hydrolysis of ATP. (2) Secondary multidrug transporters mediate the extrusion of drugs from the cell in antiport with protons (or sodium ions). The driving force for drug extrusion via proton-dependent drug antiport systems is supplied by the proton motive force, which is generated via proton extrusion by (3) the $\mathrm{F}_{1} \mathrm{~F}_{0} \mathrm{H}^{+}$-ATPase, and/or (4) redox reaction-coupled primary $\mathrm{H}^{+}$expulsion by the respiratory chain. IN and OUT refer to the inside and outside of the cytoplasmic membrane, respectively. ABC: ATP-binding cassette

cells. The structure and function of multidrug transporters is conserved from bacteria to man. On the basis of bioenergetic and structural criteria, multidrug transport systems can be divided into two major classes (Figure 1). ATP-binding cassette (ABC) transporters utilize the release of phosphate bond-energy by ATP hydrolysis, to pump drugs out of the cell (10). Secondary transporters mediate the extrusion of drugs from the cell in a coupled exchange with ions, in the absence of a chemical reaction (11). The driving force for transport is supplied by transmembrane electrochemical gradient of protons or sodium ions. In these latter cases, the transport systems are indicated as proton and sodium motive force-driven, respectively.

This paper represents a comprehensive review in which the current state of knowledge on three major aspects of drug efflux-based multidrug resistance in prokaryotic and eukaryotic cells will be summarized: (i) the functional and structural similarities among secondary and ABC-type multidrug transporters, (ii) the molecular mechanism of these transporters, and (iii) their potential physiological role.

\section{SECONDARY MULTIDRUG TRANSPORTERS}

The number of secondary multidrug transporters discovered in prokaryotic and eukaryotic cells is vast and rapidly expanding. Secondary multidrug transporters have been detected in pathogenic yeasts such as Candida albicans (caMDR1p) (12), and pathogenic bacteria such as methicillin-resistant Staphylococcus aureus (QacC) (13), Mycobacterium smegmatis (LfrA) (14), and Neisseria gonorrhoeae (MtrD) (15). Secondary multidrug transporters have also been detected in mammals, such as in rat kidney cells (OCT1) (16).

Computer-based sequence analyses have revealed that secondary drug transporters belong to one of three distinct families of transport proteins: the Major Facilitator Superfamily (MFS) (17), the ResistanceNodulation-Cell Division (RND) family (18), and the 
Table 1. Examples of secondary multidrug transporters in microorganisms.

\begin{tabular}{llll}
\hline \hline Transporter Family & Protein & Organism & Accession No. $^{\text {a }}$ \\
\hline MFS family & caMDR1p & Candida albicans & SW P28873 \\
& LmrP & GB X89779 \\
& Bmr & Lactococcus lactis & SW P33449 \\
& Qra & Bacillus subtilis & SW P21191 \\
& Emr X X & Staphylococcus aureus & SW P27304 \\
& AcrB & Escherichia coli & EM U00734 \\
RND family & MexB & Escherichia coli & GB L11616 \\
& MtrD & Pseudomonas aeruginosa & P43505 \\
& QacC & Neisseria gonorrhoeae & SW P14319 \\
SMR family & QacE & Staphylococcus aureus & PR S25583 \\
& EmrE & Klebsiella aerogenes & SW P23895 \\
\hline \hline
\end{tabular}

a Accession number: GB, Genbank; SW, SwissProt; EM, EMBL; PR, PIR. MFS: Major Facilitator Superfamily (17); RND: ResistanceNodulation-Cell Division family (18); SMR: Small Multidrug Resistance family (19).

Small Multidrug Resistance (SMR) family (19) (Table 1). Bacterial examples of each type of secondary drug transporter are Lactococcus lactis LmrP (MFS family) (20), S. aureus QacC (SMR family) (13), and Pseudomonas aeruginosa MexB (RND family) (21). LmrP and QacC have specificity for an exceptionally wide range of amphiphilic, cationic drugs including antibiotics, quaternary ammonium compounds, aromatic dyes, and phosphonium ions. MexB confers resistance to amphiphilic anionic compounds such as antibiotics, basic dyes and detergents. In Gram-negative bacteria, transport of drugs from the interior of the cell to the external medium requires the translocation of solutes across the cytoplasmic and outer membrane. Therefore, some drug transporters (e.g., MexB) in such organisms are found in association with an accessory protein which spans the periplasmic space and interacts with a porin in the outer membrane. These auxiliary proteins belong to the Membrane Fusion Protein family (18) and Outer Membrane Factor family (22).

\section{Energetics of Drug Transport}

Bacterial secondary transporters are located in the cytoplasmic membrane across which a proton motive force (interior negative and alkaline) and sodium motive force (interior negative and low) exists (Figure 1). Based on (i) the structural similarity between secondary drug transport proteins and known proton or sodium motive force-dependent transport systems, and (ii) the sensitivity of drug transport to agents that dissipate the proton and sodium motive force, it is assumed that secondary drug transporters function as drug/ion antiporters. The direct involvement of the proton motive force as driving force was demonstrated for the E. coli tetracycline transporter TetA(B) (MFS family), which mediates an electroneutral exchange reaction of a metal ${ }^{2+}$-tetracycline ${ }^{1-}$ complex with one proton (23), and L. lactis LmrP (MFS family) (24) and E. coli EmrE (SMR family) (25) which both mediate an electrogenic drug $/ n \mathrm{H}^{+}$antiport reaction $\left(\begin{array}{ll}n & 2\end{array}\right)$.

\section{Structure-Function Relationships}

Analysis of the topography of secondary drug transporters suggests the presence of either 12 or 14 membrane-spanning segments in members of the MFS family, and 12 membrane-spanning segments in members of the RND family. The transmembrane segments are most likely in $\alpha$-helical configuration, and are connected by hydrophilic loops protruding into the cytoplasm or periplasmic space. The structure of TetA(B) has been studied most extensively by limited proteolysis (26), site-directed antibody binding (27), PhoA-fusion analysis (28), and site-directed chemical labeling (29). A relatively large cytoplasmic loop in the middle of the protein separates TetA(B) in an N- and Cterminal half, each containing 6 transmembrane segments. Both halves are evolutionarily related, presumably by a gene duplication event, and are well conserved among TetA proteins of various classes (30). The $\mathrm{N}$ - and C-terminal halves of $\operatorname{Tet} \mathrm{A}(\mathrm{B})$ represent separate domains in the drug transporter (31). The functional interaction between these domains is suggested by (i) the intragenic complementation between mutations in the first and second halves of the tet $\mathrm{A}(\mathrm{B})$ gene (32), (ii) the ability of hybrid tetracycline efflux transporters containing $\mathrm{N}$ - and $\mathrm{C}$-terminal halves of different classes of tetracycline efflux proteins to confer resistance (33) and (iii) the functional reconstitution of tetracycline resistance upon coexpression of the $\mathrm{N}$ - and $\mathrm{C}$-terminal halves of TetA(B) 
as separate polypeptides (34). Members of the SMR family contain four putative transmembrane $\alpha$-helices. This topological model has recently been confirmed for QacC by genetic fusion using alkaline phosphatase and $\beta$-galactosidase as reporters of subcellular localization (35), and for E. coli EmrE by transmission fourier-transform infrared spectroscopy (36). In view of the 12 transmembrane models proposed for members of the MFS and RND families, members of the SMR family may function as a homotrimer.

Multiple alignment of the amino acid sequences of the MFS family members reveals the presence of two conserved sequence motifs that are located at similar positions within the putative secondary structure of the proteins. Motif A $(\mathrm{G} X X X \mathrm{DR} X \mathrm{GR}(\mathrm{K} / \mathrm{R}))$ is found in many members of the MFS family (17) and is present in the cytoplasmic loop between transmembrane segment 2 and 3. Motif A may be of structural importance by mediating opening and closing of the translocation pathway. Motif B (GXXXGXXGG), the drug extrusion consensus sequence, is found at the end of transmembrane segment 5, and is typical for LmrP and other drug export systems of the Major Facilitator superfamily (20,37). At present, the role of motif B in drug recognition or binding is unclear.

It has been suggested that amino residues within transmembrane segments of the human multidrug resistance P-glycoprotein MDR1 (Pgp) play a role in drug specificity (38). Observations on secondary drug transporters are consistent with this notion. The MFS members QacA and QacB in S. aureus are homologous proteins (98\% identical amino acid residues). QacB confers resistance to monovalent organic cations but differs from QacA by conferring no resistance to the divalent organic cations pentamidine isethionate and propamidine isethionate. This phenotypic difference between QacA and QacB is solely due to the presence of the acidic Asp323 within the putative transmembrane segment 10 of the QacA protein, compared to the neutral Ala323 in QacB (39). The ability of the MFS member $\mathrm{Bmr}$ in Bacillus subtilis to interact with reserpine and other drugs is strongly affected by substitutions of Val286 (40), and of Phe143 or Phe306 (41), which are located within transmembrane segments. Thus, neutral, charged and aromatic residues located within the phospholipid bilayer appear to interact with drugs and may be involved in drug recognition or binding. Interestingly, it has been shown that quaternary ammonium compounds and other cations can bind to the $\pi$ face of the aromatic ring structures of tyrosine, phenylalanine or tryptophan residues in the hydrophobic environment of the membrane $(42,43)$.

\section{ABC-TYPE MULTIDRUG TRANSPORTERS}

Transport proteins belonging to the ABC transporter superfamily are involved in the tolerance to a wide diversity of cytotoxic agents in both prokaryotes and eukaryotes (10). Included in this superfamily are the human multidrug resistance P-glycoprotein (Pgp) (44) and human multidrug resistance-associated protein MRP1 (MRP) (45) plasma membrane transporters, which catalyze the extrusion of anti-tumor drugs during the chemotherapy of cancer cells. Based on (i) the alignment analysis of amino acid residues that comprise the nucleotide binding domain(s) of ABC proteins, (ii) protein topology, and (iii) transport mechanism, the ABC transporter superfamily can be divided into two major clusters: the Pgp cluster and MRP cluster.

\section{Pgp Cluster}

Members of the Pgp cluster play an important role in microbial resistance to neutral or positively charged, amphiphilic drugs (Table 2). These transporters function in human pathogens such as the malaria parasite Plasmodium faliciparum (pfMDR1) (46), the Entamoeba histolytica (ehPgp) protozoan responsible for human amoebiasis (47), or the Leishmania donovani (ldMDR1) protozoan responsible for visceral leishmaniasis (48). In addition, infections in immunodeficient patients are often caused by the pathogenic yeast Candida albicans which expresses Cdr1p, an ABC transporter which confers antifungal resistance (49). ABC proteins in the non-pathogenic Saccharomyces cerevisiae include the multidrug transporters Pdr5p (50) and Snq2p (51). Pgp cluster members have also been identified in bacteria. In Streptomyces strains, dedicated transporters such as DrrAB $(52,53)$ mediate the excretion of specific antibiotics to ensure self-resistance to the antibiotics that they produce. A true prokaryotic multidrug transporter with significant sequence identity to Pgp in both the $\mathrm{ABC}$ and membrane domains has been found in L. lactis (LmrA) (54).

Like Pgp, some ABC transporters in microorganisms are expressed as single multifunctional polypeptides containing two homologous halves, each with an ABC domain and a membrane domain. The membrane domain is usually composed of six putative $\alpha$-helical transmembrane segments. Other ABC proteins, such as lactococcal LmrA and the Escherichia coli $\alpha$-hemolysine transporter HlyB (55), are half the size of Pgp with only a single transmembrane domain and $\mathrm{ABC}$ domain (Figure 2). The notion that two of these half-molecules must cooperate to form a single transporter is supported by the observation, amongst others, that the independent expression of each half of the $a$-mating pheromone 
Table 2. Examples of ABC-type multidrug transporters in microorganisms.

\begin{tabular}{|c|c|c|c|}
\hline & Protein & Organism & Accession No. ${ }^{a}$ \\
\hline \multirow[t]{7}{*}{ Pgp cluster } & LmrA & Lactococcus lactis & GB U63741 \\
\hline & pfMDR1 & Plasmodium falciparum & GB A32547 \\
\hline & ehPgp & Entamoeba histolytica & GB M88599 \\
\hline & ldMDR1 & Leishmania donovani & GB L01572 \\
\hline & Cdr1p & Candida albicans & GB X77589 \\
\hline & Pdr5p & Saccharomyces cerevisiae & GB L19922 \\
\hline & Snq2p & Saccharomyces cerevisiae & GB Z48008 \\
\hline \multirow[t]{4}{*}{ MRP cluster } & Ycf1p & Saccharomyces cerevisiae & GB Z48179 \\
\hline & Yor1p & Saccharomyces cerevisiae & GB Z73066 \\
\hline & ltPgpA & Leishmania tarentolae & GB A34207 \\
\hline & ceMRP1 & Caenorhabditis elegans & EM 466260 \\
\hline
\end{tabular}

a Accession number: GB, Genbank; EM, EMBL. ABC: ATP-binding cassette superfamily (10)

transporter Ste6p in yeast cells does not yield a functional transporter, while simultaneous expression of both halves does (56). In drug transporters such as DrrAB, the two membrane domains are fused into a single polypeptide which is associated with a second polypeptide containing the two ABC domains.

Interestingly, the sequence conservation between LmrA and Pgp includes particular regions (e.g., the first cytoplasmic loop and the region comprising transmembrane segments $\mathrm{V}$ and VI) that have been implicated as determinants of drug recognition and binding by Pgp. LmrA also shares significant sequence identity with the hop-resistance protein HorA in Lactobacillus brevis (57) and ABC proteins in Bacillus subtilis, Staphylococcus aureus, Escherichia coli, Helicobacter pylori, Haemophilus influenzae, and Mycoplasma genitalium (58).

\section{MRP Cluster}

Members of the MRP cluster contain an N-terminal membrane-bound domain consisting of five $\alpha$-helical transmembrane segments, which is followed by two homologous halves each with six $\alpha$-helical transmembrane segments and an ABC domain (59). Like MRP, MRP homologs in microorganisms mediate the transport of a wide variety of organic anions, such as carboxyfluorescein derivatives, taurocholate, bis(glutathionate)-cadmium and other glutathione $\mathrm{S}$-conjugates. The best characterized members of this cluster include: (i) the yeast cadmium factor (Ycf1) $(60,61)$ and Yor1 protein (62) in S. cerevisiae, (ii) transporters associated with heavy metal resistance in Leishmania species ( $\operatorname{lmPgpA})(63,64)$ and the nematode Caenorhabditis elegans (ceMRP1) (65), and (iii) the bile acid transporter Bat1p in S. cerevisiae (66) (Table 2). The BCECF (2',7'-bis-(2-carboxyethyl)-5(and 6)carboxyfluorescein) transporter in L. lactis may be the first prokaryotic example of this cluster (67).

\section{Structure-Function Relationships}

The effects of amino acid substitutions on the substrate specificity of ABC-type multidrug transporters has been studied most extensively using Pgp. In general, Pgp mutants can be classified into three groups. The first group is of mutations Gly141-to-Val, Gly185-to-Val, and Gly187-to-Val, and others, all in the first cytoplasmic loop (68-70); Gly228-to-Val in the second cytoplasmic loop (71); Gly812-to-Val and Gly830-to-Val in transmembrane segment 4 (71); Phe335-to-Ala and Val338-to-Ala in transmembrane segment $6(72,73)$. These mutations increase the ability of $\mathrm{Pgp}$ to confer resistance to colchicine and doxorubicin, and decrease the ability of Pgp to confer resistance to vinblastine. The second group consists of mutations Pro223-to-Ala in transmembrane segment 4 (74); Gly341-to-Val in transmembrane segment 6 (73); Pro866-to-Ala in transmembrane segment 10 (74); Phe978-to-Ala in transmembrane segment 12 (72); Ser939-to-Phe, Tyr949-to-Ala, and Phe953-to-Ala in transmembrane segment 11 of mouse mdr1 $(75,76)$; and Ser941-to-Phe in transmembrane segment 11 of mouse mdr3 (77). These mutations decrease the ability of Pgp to confer resistance to colchicine and doxorubicin, and increase or do not affect the ability of Pgp to confer resistance to vinblastine. Interestingly, Pgp mutants with amino acid substitutions at position 61 in transmembrane segment 1 fall into the first or the second group of mutants, depending on the size of the side chain (78). Hence, this transmembrane segment is also important in substrate specificity. The third group of mutations that affect substrate specificity of Pgp fall in the ATP-binding domains: Lys536-to-Arg in the ABC signature sequence, and mutations at positions 522-525 and 578 near the Walker B region of the $\mathrm{N}$ terminal ATP-binding domain $(79,80)$. In summary, amino acid substitutions which affect the substrate specificity of Pgp are found scattered throughout the 


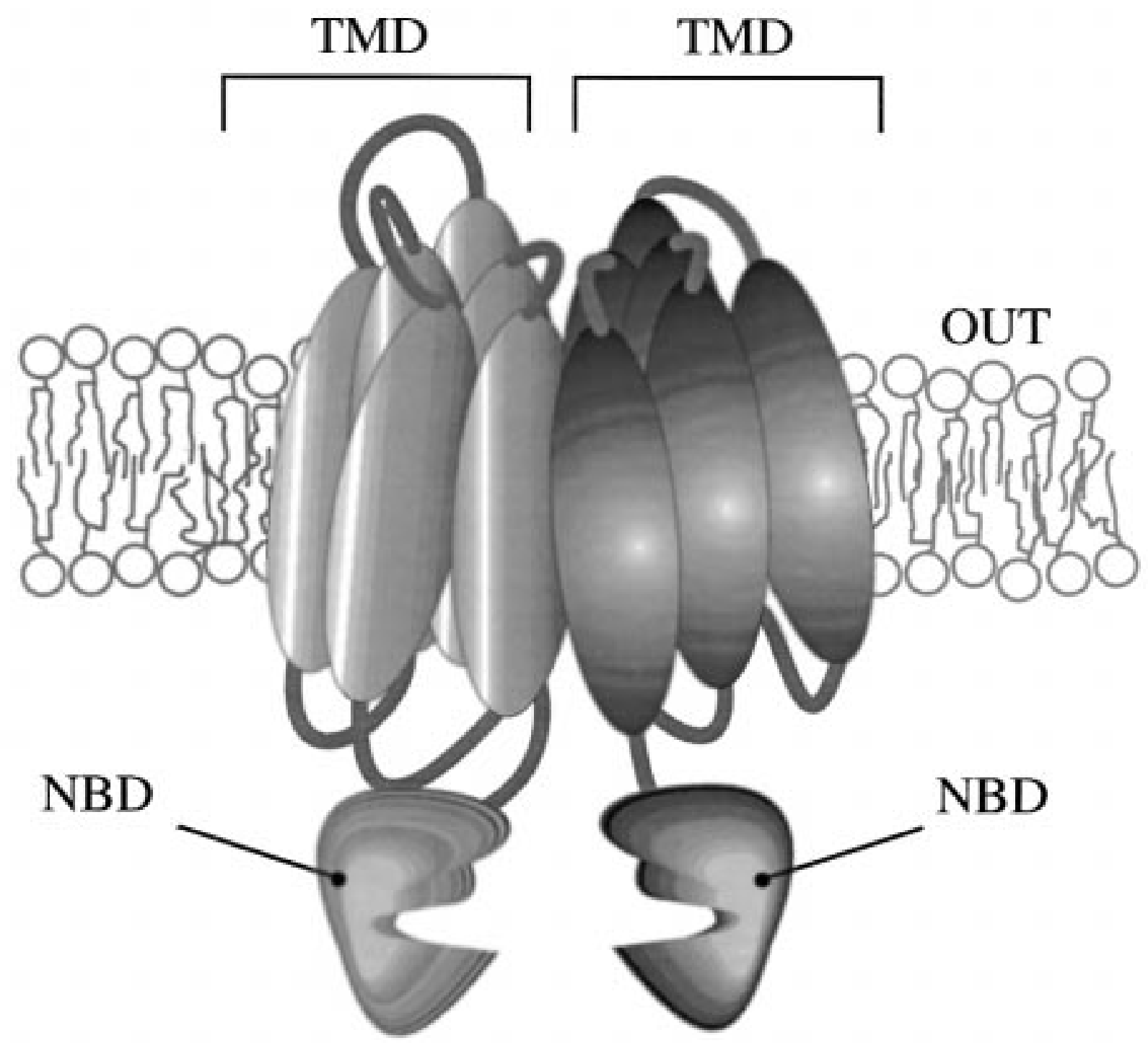

Figure 2. Structural model of a homodimer of the ABC-type multidrug transporter LmrA of Lactococcus lactis. LmrA is predicted to contain a transmembrane domain (TMD) with six transmembrane $\alpha$-helices (depicted as ellipses), and a nucleotide-binding domain (NBD) with the ABC signature and Walker A/B sequences (10). The structure of LmrA is very similar to that of the mammalian multidrug resistance P-glycoprotein (Pgp). Pgp contains two covalently linked homologous halves, each with 6 transmembrane segments and an ATP-binding domain. In view of the twelve-transmembrane model proposed for Pgp, LmrA may function as a homodimeric complex. IN and OUT refer to the inside and outside of the cytoplasmic membrane, respectively.

molecule in transmembrane domains, cytoplasmic loops and ATP binding domains.

A limited set of chimeric Pgp proteins has been constructed using mammalian P-glycoproteins, such as human MDR2, as partner protein to identify proteins segments and amino acids in Pgp implicated in drug recognition (81-83). Human MDR2 (often called MDR3), a phosphatidylcholine-specific translocase in the bile canicular membrane of hepatocytes, shares about $77 \%$ identity with Pgp. However, overexpression of human MDR2 or mouse mdr2 in drug-sensitive cells does not confer MDR (84,85). Greenberger and coworkers have replaced regions with the greatest sequence diversity between $\mathrm{Pgp}$ and MDR2, from transmembrane segment 10 to the C-terminus of Pgp, by the corresponding sequences from MDR2 (83). Only a replacement limited to transmembrane segment 12 of both proteins results in a functional hybrid Pgp, which was markedly impaired in conferring resistance to actinomycin D, vincristine, and doxorubicin, but not to colchicine. The drug resistance phenotype was associated with an impaired ability to photoaffinity label the hybrid protein with iodoaryl azidoprazosin. Thus, amino acids within transmembrane segment 12 may 


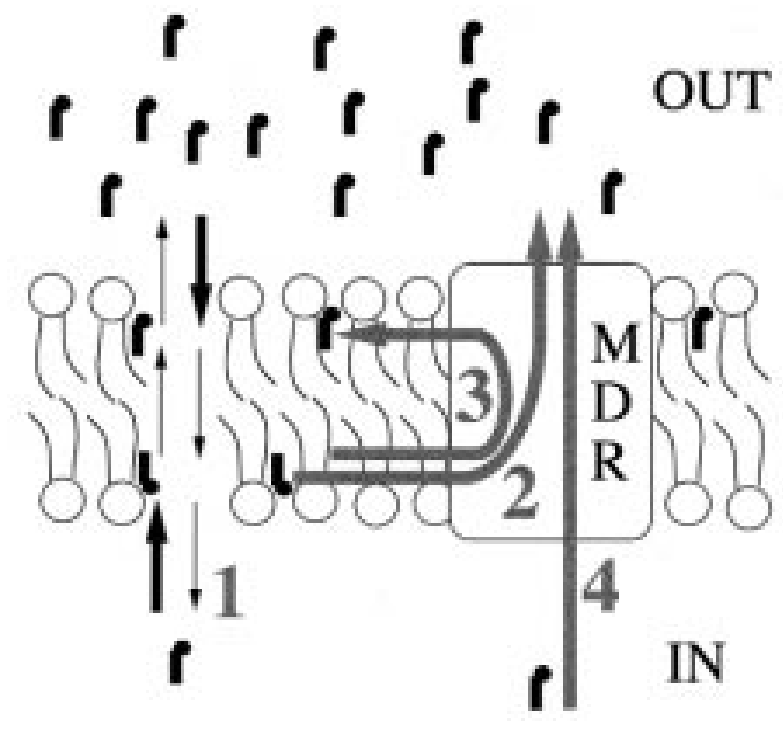

Figure 3. Possible mechanisms of drug transport across the cytoplasmic membrane. (1) Drugs enter the cell by passive diffusion via the outer and inner leaflet of the phospholipid bilayer. Due to their lipophilicity, a significant amount of drug molecules will be retained in the membrane. Therefore, drugs may be expelled from the cell through: (2) extrusion from the inner leaflet to the external water phase (hydrophobic vacuum cleaner model), and/or (3) extrusion from the inner leaflet of the phospholipid bilayer to the outer leaflet (flippase model). (4) Alternatively, a drug molecule may be moved back to extracellular environment by extrusion from the internal water phase to the external water phase (aqueous pore model). Partitioning of drugs between the water phase and phospholipid bilayer is indicated by a black arrow. Protein-mediated drug transport is indicated by a gray arrow. IN and OUT refer to the inside and outside of the cytoplasmic membrane, respectively. MDR: multidrug resistance protein

compose part of a drug binding pocket of Pgp and are in close proximity to a photoaffinity drug-labeling domain.

Taken together, a number of amino acid substitutions in Pgp have been found to affect the drug specificity of the protein, but particular domains in Pgp involved in drug recognition, binding and translocation have not yet been identified.

\section{Functional Complementation}

The structural similarity between ABC-type drug transporters can result in functional similarity as the mouse Pgp-homolog mdr3 (86) and Plasmodium pfMdr1 (87) can complement yeast Ste6p, thus restoring mating in a $\Delta$ ste 6 sterile yeast strain. Likewise, MRP can functionally complement the Ycf1 protein in a $\Delta y c f l$ cadmium-sensitive strain of $S$. cerevisiae (88), and partially complement Ste6p in the sterile $\Delta$ ste6 null mutant of this organism (89). The functional substitution of one ABC-type drug transporter by another is not only confined to eukaryotic transport proteins. Bacterial LmrA was able to functionally complement human Pgp in human lung fibroblast cells (90). Surprisingly, LmrA was targeted to the plasma membrane. The pharmacological characteristics of LmrA and Pgp-expressing lung fibroblasts were very similar, and the affinities of both proteins for vinblastine and magnesium-ATP indistinguishable. Blockers of P-glycoprotein-mediated multidrug resistance also inhibited LmrA-dependent drug resistance. Kinetic analysis of drug dissociation from LmrA expressed in plasma membranes of insect cells, revealed the presence of two allosterically-linked drug binding sites indistinguishable from those of Pglycoprotein $(90,91)$. The remarkable conservation of function between ABC-type drug transporters suggests a common fundamental molecular mechanism of these proteins in prokaryotic and eukaryotic cells.

\section{MOLECULAR MECHANISM}

Several transport models have been postulated for Pgp and LmrA pump function to explain the broad specificity for chemically unrelated compounds (Figure 3). Drug translocation may involve substrate transport from the cytoplasm to the exterior (conventional transport hypothesis (92)) which would require an enormous flexibility of an 'enzyme-like' substrate recognition site. Alternatively, the multidrug transporters could recognize the lipophilic drugs by their physical property to intercalate into the lipid bilayer, and transport drugs from the lipid bilayer to the exterior (vacuum cleaner hypothesis (93)), or from the inner leaflet to the outer leaflet of the lipid bilayer (flippase hypothesis (94)). A property common to all known Pgp and LmrA substrates is their ability to intercalate between the phospholipids of biological membranes. This notion has led to the suggestion that these transporters recognize substrates within the membrane $(24,44,95)$. Drug recognition within the membrane is supported by a number of observations: (i) photoaffinity analogs of Pgp-substrates label Pgp predominantly in or near the transmembrane helices 4-6, and 11-12 (96-98), (ii) point mutations resulting in the alteration in drug specificity of Pgp are frequently found in transmembrane helices (38), (iii) acetoxymethyl esters of several fluorescent probes accumulate less in Pgp or LmrA-expressing cells, despite the fact that the ester moieties are rapidly cleaved by intracellular esterases and the resulting carboxylates are not substrates for Pgp and $\operatorname{LmrA}(95,99)$, (iv) the kinetics of ATP-dependent transport of Hoechst 33342 by Pgp and of TMA-DPH by LmrA in membrane vesicles is consistent with transport of the compounds from the inner, but not from the outer leaflet of the lipid bilayer $(95,100,101)$. Interestingly, these latter observations have also been made for the secondary lactococcal multidrug transporter LmrP (24). Thus, a main determinant of specificity would be the 
ability of a substrate to be intercalated into the lipid bilayer.

This transport mechanism is likely to be a more general mechanism for $\mathrm{ABC}$ transporters with hydrophobic substrates. The human MDR2 geneencoded P-glycoprotein transports phosphatidylcholine from the cytoplasmic leaflet of the bile canicular membrane of hepatocytes into the bile $(84,102)$. In addition, the $E$. coli $\alpha$-hemolysine transporter HlyB most likely binds the transport signal sequence of $\alpha$-hemolysine, when the signal sequence forms an amphiphilic helix that binds to the cytoplasmic leaflet of the plasma membrane $(103,104)$.

\section{PHYSIOLOGICAL ROLE}

It has been proposed that Pgp participates in the protection of human cells against hydrophobic xenobiotics by active excretion of these compounds from the membrane into bile, urine, or the intestinal lumen, preventing their accumulation in critical organs such as the brain (105). Likewise, a defense function can be envisaged for multidrug transporters in microorganisms. These organisms encounter numerous hydrophobic compounds in their habitat which will accumulate in phospholipid bilayers (for review see 106). It is noteworthy that the natural environment of enteric microorganisms is enriched in bile salts and fatty acids, and that these compounds are substrates for various multidrug transporters $(107,108)$. In addition, some microbial multidrug transporters may play a role in the transport of endogenous substrates, such as lipids $(84,102,109,110)$, pheromones $(111,112)$, or lipid-linked precursors of peptidoglycan (for review see (113)) or other extracellular biopolymers.

\section{CONCLUSION}

Infectious disease caused by multidrug resistant microorganisms is an increasingly complex clinical and public health problem. Prevention and control strategies will require the application of epidemiological and behavioral approaches, as well as research technologies aimed at the basic mechanisms of drug resistance. This review summarizes the molecular properties of multidrug transporters in prokaryotic and eukaryotic cells, and shows that the microbial systems are very similar in structure and function to those found in mammalian tumor cells. Interestingly, multidrug transporters have an exceptionally broad drug specificity, and can shift their specificities in the course of drug treatment. The structure-function relationships that dictate drug recognition by multidrug transporters will represent an intriguing new area of inquiry, and may allow the rational development of inhibitors of these proteins, and of new drugs which are not extruded from the cell.

\section{ACKNOWLEDGMENTS}

The authors would like to thank Marloes Veenstra for excellent technical assistance. Research in the authors' laboratory was supported by the University of Groningen, the Biotechnology program of the Commission of the European Communities, the Dutch Cancer Society. H.W.V.V. is a fellow of the Royal Netherlands Academy of Arts and Sciences.

\section{REFERENCES}

1. Hughes JM, Tenover FC. Approaches to limiting emergence of antimicrobial resistance in bacteria in human populations. Clinical Infectious Diseases 24: S131-S135; 1997.

2. Levy SB. The challenge of antibiotic resistance. Scientific American 278: 46-53; 1998.

3. Perreten V, Schwarz F, Cresta L, et al. Antibiotic resistance spread in food. Nature 389: 801-802; 1997.

4. Borst P, Ouellette M. New mechanisms of drug resistance in parasitic protozoa. Annual Review of Microbiology 49: 427460; 1995.

5. Spratt BG. Resistance to antibiotics mediated by target alterations. Science 264: 388-393; 1994.

6. Davies J. Inactivation of antibiotics and the dissemination of resistance genes. Science 264: 375-382; 1994.

7. Nikaido H. Prevention of drug access to bacterial targets: permeability barriers and active efflux. Science 264: 382-388; 1994.

8. Lewis K. Multidrug resistance pumps in bacteria: variations on a theme. Trends in Biochemical Sciences 19: 119-123; 1994.

9. Balzi E, Goffeau A. Genetics and biochemistry of yeast multidrug resistance. Biochimica et Biophysica Acta 1187: 152-162; 1994.

10. Higgins CF. ABC transporters: from microorganisms to man. Annual Review of Cell Biology 8: 67-113; 1992.

11. Paulsen IT, Brown MH, Skurray RA. Proton-dependent multidrug efflux systems. Microbiological Reviews 60: 575608; 1996.

12. Ben-Yaacov R, Knoller S, Caldwell GA, et al. The Candida albicans gene encoding resistance to benomyl and methotrexate is a multidrug resistance gene. Antimicrobial Agents and Chemotherapy 38: 648-652; 1994.

13. Littlejohn TG, Paulsen IT, Gillespie MT, et al. Substrate specificity and energetics of antiseptic and disinfectant resistance in Staphylococcus aureus. FEMS Microbiology Letters 95: 259-266; 1992.

14. Liu J, Takiff HE, Nikaido H. Active efflux of fluoroquinolones in Mycobacterium smegmatis mediated by LfrA, a multidrug efflux pump. Journal of Bacteriology 178: 3791-3795; 1997.

15. Hagman KE, Pan W, Spratt BG, et al. Resistance of Neisseria gonorrhoeae to antimicrobial agents is modulated by the mtrRCDE efflux system. Microbiology 141: 611-622; 1995.

16. Gründemann D, Gorboulev V, Gambaryan S, et al. Drug excretion mediated by a new prototype of polyspecific transporter. Nature 372: 549-552; 1994.

17. Marger M, Saier MH. A major superfamily of transmembrane facilitators that catalyze uniport, symport and antiport. Trends in Biochemical Sciences 18: 13-20; 1993.

18. Saier MH, Tam R, Reizer A, et al. Two novel families of bacterial membrane proteins concerned with nodulation, cell division and transport. Molecular Microbiology 11: 841-847; 1994.

19. Paulsen IT, Skurray RA, Tam R, et al. The SMR family: a novel family of multidrug efflux proteins involved with the efflux of lipophilic drugs. Molecular Microbiology 19: 1167-1175; 1996.

20. Bolhuis H, Poelarends G, van Veen HW, et al. The lactococcal $\operatorname{lmr} P$ gene encodes a proton motive force-dependent drug transporter. 
Journal of Biological Chemistry 270: 26092-26098; 1995.

21. Poole K, Krebes K, McNally C, Neshat, S. Multiple antibiotic resistance in Pseudomonas aeruginosa evidence for involvement of an efflux operon. Journal of Bacteriology 175: 7363-7372; 1993.

22. Dinh T, Paulsen IT, Saier MH. A family of extracytoplasmic proteins that allow transport of large molecules across the outer membranes of gram-negative bacteria. Journal of Bacteriology 176: 3825-3831; 1994.

23. Yamaguchi A, Iwasaki-Ohba Y, Ono N, et al. Stoichiometry of metal-tetracycline $/ \mathrm{H}^{+}$antiport mediated by transposon $\mathrm{Tn} 10$ encoded tetracycline resistance protein. FEBS Letters 282: 415$418 ; 1991$.

24. Bolhuis H, van Veen HW, Brands JR, et al. Energetics and mechanism of drug transport mediated by the lactococcal MDR transporter LmrP. Journal of Biological Chemistry 271: 2412324128; 1996.

25. Yerushalmi H, Lebendiker $\mathrm{M}$, Schuldiner S. EmrE, an Escherichia coli $12-\mathrm{kDa}$ multidrug transporter, exchanges toxic cations and $\mathrm{H}^{+}$and is soluble in organic solvents. Journal of Biological Chemistry 270: 6856-6863; 1995.

26. Eckert B, Beck CF. Topology of the transposon Tn10-encoded tetracycline resistance protein within the inner membrane of Escherichia coli. Journal of Biological Chemistry 264: 1166311670; 1989.

27. Yamaguchi A, Adachi K, Sawai T. Orientation of the carboxyl terminus of the transposon Tn10-encoded tetracycline resistance protein. FEBS Letters 265: 17-19; 1990.

28. Allard JD, Bertrand KP. Membrane topology of the pBR322 tetracycline resistance protein. Journal of Biological Chemistry 267: 17809-17819; 1992.

29. Kimura T, Ohnuma M, Sawai T, Yamaguchi. A. Membrane topology of the transposon 10-encoded metal-tetracycline $/ \mathrm{H}^{+}$ antiporter as studied by site-directed chemical labeling. Journal of Biological Chemistry 272: 580-585; 1997.

30. Rubin RA, Levy SB, Heinrikson RL, Kezdy FS. Gene duplication in the evolution of the two complementing domains of gramnegative tetracycline efflux proteins. Gene 87: 7-13; 1990.

31. Curiale M, Levy SB. Two complementation groups mediate tetracycline resistance determined by Tn10. Journal of Bacteriology 151: 209-215; 1982.

32. McNicholas P, McGlynn M, Guay GG, Rothstein DM. Genetic analysis suggests functional interactions between the $\mathrm{N}$ - and $\mathrm{C}$ terminal domains of the TetA(C) efflux pump encoded by pBR322. Journal of Bacteriology 177: 5355-5357; 1995.

33. Rubin RA, Levy SB. Interdomain hybrid Tet proteins confer tetracycline resistance only when derived from more closely related members of the tet gene family. Journal of Bacteriology 172: 2303-2312; 1990.

34. Rubin RA, Levy SB. Tet protein domains interact productively to mediate tetracycline resistance when present on separate polypeptides. Journal of Bacteriology 173: 4503-4509; 1991.

35. Paulsen IT, Brown MH, Dunstan SJ, Skurray RA. Molecular characterization of the staphylococcal multidrug resistance export protein QacC. Journal of Bacteriology 177: 2827-2833; 1995.

36. Arkin IT, Russ WP, Lebendiker M, Schuldiner S. Determining the secondary structure and orientation of EmrE, a multidrug transporter, indicates a transmembrane four-helix bundle. Biochemistry 35: 7233-7238; 1996.

37. Griffith JK, Baker ME, Rouch DA, et al. Membrane transport proteins: implications of sequence comparisons. Current Opinion in Cell Biology 4: 684-695; 1992.

38. Gottesman MM, Hrycyna CA, Schoenlein PV, et al. Genetic analysis of the multidrug transporter. Annual Review of Genetics. 29: 607-649; 1995.

39. Paulsen IT, Brown MH, Littlejohn TG, et al. Multidrug resistance proteins QacA and QacB from Staphylococcus aureus: membrane topology and identification of residues involved in substrate specificity. Proceedings of the National Academy of Sciences (USA) 93: 3630-3635; 1996.

40. Ahmed M, Borsch CM, Neyfakh AA, Schuldiner S. Mutants of the Bacillus subtilis multidrug transporter Bmr with altered sensitivity to the antihypertensive alkaloid reserpine. Journal of Biological Chemistry 268: 11086-11089; 1993.

41. Klyachko KA, Schuldiner S, Neyfakh AA. Mutations affecting substrate specificity of the Bacillus subtilis multidrug transporter Bmr. Journal of Bacteriology 179: 2189-2193; 1997.

42. Dougherty DA. Cation-p interactions in chemistry and biology: a new view of benzene, Phe, Tyr, and Trp. Science 271: 163168; 1996.

43. Popkov M, Lussier I, Medvedkine V, et al. Multidrug-resistance drug-binding peptides generated by using a phage display library. European Journal of Biochemistry 251: 155-163; 1998.

44. Gottesman MM, Pastan I. Biochemistry of multidrug resistance mediated by the multidrug transporter. Annual Review of Biochemistry 62: 385-427; 1993.

45. Cole SPC, Bhardwaj G, Gerlach JH, et al. Overexpression of a transporter gene in a multidrug-resistant human lung cancer cell line. Science 258: 1650-1654; 1992.

46. Wilson CM, Serrano AE, Wasley A, et al. Amplification of a gene related to mammalian $\mathrm{mdr}$ genes in drug-resistant Plasmodium falciparum. Science 244: 1184-1186; 1989.

47. Descoteaux S, Ayala P, Orozco E, Samuelson J. Primary sequences of two P-glycoprotein genes in Entamoeba histolytica. Molecular and Biochemical Parasitology 54: 201212; 1992.

48. Henderson DM, Sifri CD, Rodgers M, et al. Multidrug resistance in Leishmania donovani is conferred by amplification of a gene homologous to the mammalian $m d r 1$ gene. Molecular and Cellular Biology 12: 2855-2865; 1992.

49. Prasad R, de Wergifosse P, Goffeau A, Balzi E. Molecular cloning and characterization of a novel gene of Candida albicans conferring multiple resistance to drugs and antifungals. Current Genetics 27: 320-329; 1995.

50. Balzi E, Wang M, Leterme S, et al. Pdr5, a novel yeast multidrug resistance conferring transporter controlled by the transcription regulator Pdr1. Journal of Biological Chemistry 269: 22062214; 1994

51. Decottignies A, Lambert L, Catty $\mathrm{P}$, et al. Identification and characterization of Snq2, a new multidrug ATP binding cassette transporter of the yeast plasma membrane. Journal of Biological Chemistry 270: 18150-18157; 1995.

52. Guilfoile PG, Hutchinson CR. A bacterial analog of the $m d r$ gene of mammalian tumour cells is present in Streptomyces peuceticus, the producer of daunorubicin and doxorubicin. Proceedings of the National Academy of Sciences (USA) 88: 8553-8557; 1991.

53. Méndez C, Salas JA. ABC transporters in antibiotic-producing actinomycetes. FEMS Microbiology Letters 158: 1-8; 1998.

54. van Veen HW, Venema K, Bolhuis H, et al. Multidrug resistance mediated by a bacterial homolog of the human multidrug transporter MDR1. Proceedings of the National Academy of Sciences (USA) 93: 10668-10672; 1996.

55. Felmlee T, Pellett S, Welch RA. Nucleotide sequence of an Escherichia coli chromosomal hemolysin. Journal of Bacteriology 163: 94-105; 1985.

56. Berkower C, Michaelis S. Mutational analysis of the yeast afactor transporter STE6, a member of the ATP binding cassette (ABC) protein superfamily. EMBO Journal 10: 3777-3785; 1991.

57. Sami M, Yamashita H, Hirono $T$, et al. Hop-resistant Lactobacillus brevis contains a novel plasmid harboring a 
multidrug resistance-like gene. Journal of Fermentation and Bioengineering 84: 1-6; 1997.

58. van Veen HW, Konings WN. The ABC family of multidrug transporters in microorganisms. Biochimica et Biophysica Acta 1365: 31-36; 1998.

59. Deeley RG, Cole SPC. Function, evolution and structure of multidrug resistance protein (MRP). Seminars in Cancer Biology 8: 193-204; 1997.

60. Li Z-S, Szczypka M, Lu Y-P, et al. The yeast cadmium factor protein (Ycf1) is a vacuolar glutathione S-conjugate pump. Journal of Biological Chemistry 271: 6509-6517; 1996.

61. Li Z-S, Lu Y-P, Zhen R-G, et al. A new pathway for cadmium sequestration in Saccharomyces cerevisiae: YCF-1 catalyzed transport of bis(glutathionato)cadmium. Proceedings of the National Academy of Sciences (USA) 94: 42-47; 1997.

62. Cui Z, Hirata D, Tsuchiya E, et al. The multidrug resistanceassociated protein (MRP) subfamily (Yrs1/Yor1) of Saccharomyces cerevisiae is important for the tolerance to a broad range of organic anions. Journal of Biological Chemistry 271: 14712-14716; 1996.

63. Callahan HL, Beverley SM. Heavy metal resistance: a new role of P-glycoproteins in Leishmania. Journal of Biological Chemistry 266: 18427-18436; 1991.

64. Grondin K, Haimeur A, Mukhopadhyay R, et al. Coamplification of the g-glutamylcysteine synthetase gene $g s h 1$ and of the $\mathrm{ABC}$ transporter gene pgpA in arsenite-resistant Leishmania tarentolae. EMBO Journal 16: 3057-3065; 1997.

65. Broeks A, Gerrard B, Allikmets R, et al. Homologues of the human multidrug resistance genes $M R P$ and $M D R$ contribute to heavy metal resistance in the soil nematode Caenorhabditis elegans. EMBO Journal 15: 6132-6143; 1996.

66. Ortiz DF, St Pierre MV, Abdulmessih A, Arias IM. A yeast ATPbinding cassette-type protein mediating ATP-dependent bile acid transport. Journal of Biological Chemistry 272: 15358$15365 ; 1997$.

67. Molenaar D, Bolhuis H, Abee T, et al. The efflux of a fluorescent probe is catalyzed by an ATP-driven extrusion system in Lactococcus lactis. Journal of Bacteriology 174: 3118-3124; 1992.

68. Choi K, Chen C, Kriegler M, Roninson IB. An altered pattern of cross-resistance in multidrug-resistant human cells results from spontaneaous mutations in the $m d r 1$ (P-glycoprotein) gene. Cell 53: 519-529; 1988

69. Kioka N, Tsubota J, Kakehi Y, et al. P-glycoprotein gene (MDR1) cDNA from human adrenal: normal P-glycoprotein carries Gly-185 with an altered pattern of multidrug resistance. Biochemical and Biophysical Research Communications 162: 224; 1989.

70. Kwan T, Gros P. Mutational analysis of the P-glycprotein first intracellular loop and flanking transmembrane domains. Biochemistry 37: 3337-3350; 1998 .

71. Loo TW, Clarke DM. Functional consequences of glycine mutation in the predicted cytoplasmic loops of P-glycoprotein. Journal of Biological Chemistry 269: 7243-7248; 1994.

72. Loo TW, Clarke DM. Functional consequences of phenylalanine mutations in the predicted transmembrane domains of $\mathrm{P}$ glycoprotein. Journal of Biological Chemistry 268: 1996519972; 1993.

73. Loo TW, Clarke DM. Mutation of amino acids located in predicted transmembrane domain segment 6 (TM6) modulate the activity and substrate specificity of human P-glycprotein. Biochemistry 33: 14049-14057; 1994.

74. Loo TW, Clarke DM. Functional consequences of proline mutations in the transmembrane domain of P-glycoprotein. Journal of Biological Chemistry 268: 3143-3149; 1993.

75. Hanna M, Brault $M$, Kwan $T$, et al. Mutagenesis of transmembrane domain 11 of P-glycoprotein by alanine scanning. Biochemistry 35: 3625-3635; 1996.

76. Kajiji S, Talbot F, Grizzuti K, et al. Functional analysis of Pglycoprotein mutants identifies predicted transmembrane domain 11 as a putative drug binding site. Biochemistry 32: 4185-4194; 1993.

77. Gros P, Dhir R, Croop J, Talbot F. A single amino acid substitution strongly modulates the activity and substrate specificity of the mouse mdr1 and mdr3 drug efflux pumps. Proceedings of the National Academy of Sciences (USA) 88: 7289-7293; 1991

78. Taguchi Y, Kino K, Morishima M, et al. Amino acid substitutions in the first transmembrane domain (TM1) of P-glycoprotein alter substrate specificity. Biochemistry 36: 8883-8889; 1997.

79. Hoof T, Demmer A, Hadam MR, et al. Cystic fibrosis-type mutational analysis in the ATP-binding cassette transporter of Pglycoprotein. Journal of Biological Chemistry 269: 2057520583; 1994.

80. Beaudet L, Gros P. Functional dissection of P-glycoprotein nucleotide-binding domains in chimeric and mutant proteins. Journal of Biological Chemistry 270: 17159-17170; 1995.

81. Currier SJ, Kane SE, Willingham MC, et al. Identification of residues in the first cytoplasmic loop of P-glycoprotein involved in the function of chimeric human MDR1-MDR2 transporters. Journal of Biological Chemistry 267: 25153-25159; 1992.

82. Dhir R, Gros P. Functional analysis of chimeric proteins constructed by exchanging homologous domains of two Pglycoproteins conferring distinct drug resistance profiles. Biochemistry 31: 6103-6110; 1992.

83. Zhang X, Collins KI, Greenberger LM. Functional evidence that transmembrane 12 and the loop between transmembrane 11 and 12 form part of the drug-binding domain in P-glycoprotein encoded by MDR1. Journal of Biological Chemistry 270: 54415448; 1995.

84. Smit JJM, Schinkel AH, Oude Elferink RPJ, et al. Homozygous disruption of the murine mdr2 P-glycoprotein gene leads to a complete absence of phospholipid from bile and to liver disease. Cell 75: 451-462; 1993.

85. Smith AJ, Timmermans-Hereijgers JLPM, Roelofsen B, et al. The human MDR3 P-glycoprotein promotes translocation of phosphatidyl choline through the plasma membrane of fibroblasts from transgenic mice. FEBS Letters 354: 263-266; 1994.

86. Raymond M, Gros P, Whiteway M, Thomas DY. Functional complementation of yeast ste6 by a mammalian multidrug resistance $m d r$ gene. Science 256: 232-234; 1992.

87. Volkman SK, Cowman AF, Wirth DF. Functional complementation of the ste6 gene of Saccharomyces cerevisiae with the $p f m d r l$ gene of Plasmodium falciparum. Proceedings of the National Academy of Sciences (USA) 92: 8921-8925; 1995.

88. Tommasini R, Evers R, Vogt E, et al. The human multidrug resistance-associated protein functionally complements the yeast cadmium resistance factor 1. Proceedings of the National Academy of Sciences (USA) 93: 6743-6748; 1996.

89. Ruetz S, Brault M, Kast C, et al. Functional expression of the multidrug resistance-associated protein in the yeast Saccharomyces cerevisiae. Journal of Biological Chemistry 271: 4154-4160; 1996

90. van Veen HW, Callaghan $\mathrm{R}$, Soceneantu $\mathrm{L}$, et al. A bacterial antibiotic resistance gene that complements the human multidrug resistance P-glycoprotein gene. Nature 391: 291-295; 1998.

91. Martin C, Berridge G, Higgins CF, Callaghan R. The multidrug resistance reversal agent SR33557 and modulation of vinca alkaloid binding to P-glycoprotein by an allosteric interaction. British Journal of Pharmacology 122: 765-771; 1997.

92. Altenberg GA, Vanoye CG, Horton JK, Reuss L. Unidirectional 
fluxes of rhodamine 123 in multidrug resistant cells: evidence against direct extrusion from the plasma membrane. Proceedings of the National Academy of Sciences (USA) 91: 4654-4657; 1994.

93. Raviv Y, Pollard HB, Bruggeman EP, et al. Photosensitized labeling of a functional multidrug transporter in living drug resistant tumor cells. Journal of Biological Chemistry 265: 3975-3980; 1990

94. Higgins CF, Gottesman MM. Is the multidrug transporter a flippase? Trends in Biochemical Sciences 17: 18-21; 1992.

95. Bolhuis H, van Veen HW, Molenaar D, et al. Multidrug resistance in Lactococcus lactis: evidence for ATP-dependent drug extrusion from the inner leaflet of the cytoplasmic membrane. EMBO Journal 15: 4239-4245; 1996.

96. Greenberger LM. Major photoaffinity drug labeling sites for iodoaryl azidoprazosin in P-glycoprotein are within, or immediately C-terminal to, transmembrane domains 6 and 12 . Journal of Biological Chemistry 268: 11417-11425; 1993.

97. Morris DI, Greenberger LM, Bruggeman EP, et al. Localization of the forskolin labeling sites for both halves of P-glycoprotein: similarity of the sites labeled by forskolin and prazosin. Molecular Pharmacology 46: 329-337; 1994.

98. Germann UA. P-glycoprotein - a mediator of multidrug resistance in tumour cells. European Journal of Cancer 32A: 927-944; 1996.

99. Homolya L, Holló Z, Germann UA, et al. Fluorescent cellular indicators are extruded by the multidrug resistance protein. Journal of Biological Chemistry 268: 21493-21496; 1993.

100. Shapiro AB, Ling V. P-glycoprotein-mediated Hoechst 33342 transport out of the lipid bilayer. European Journal of Biochemistry 250: 115-121; 1997.

101. Shapiro AB, Ling V. Extraction of Hoechst 33342 from the cytoplamsic leaflet of the plasma membrane by P-glycoprotein. European Journal of Biochemistry 250: 122-129; 1997.

102. Ruetz S, Gros P. Phosphatidylcholine translocase: a physiological role for the mdr2 gene. Cell 77: 1071-1081; 1994.

103. Zhang F, Yin Y, Arrowsmith CH, Ling V. Secretion and circular dichroism analysis of the C-terminal signal peptides of HlyA and LktA. Biochemistry 34: 4193-4201; 1995.

104. Sheps JA, Cheung I, Ling V. Hemolysin transport in Escherichia coli: point mutations in HlyB compensate for a deletion in the predicted amphiphilic helix regions of the HlyA signal. Journal of Biological Chemistry 270: 14829-14834; 1995.

105. Schinkel AH, Smit JJ, van Tellingen O, et al. Disruption of the mouse mdrla P-glycoprotein gene leads to a deficiency in the blood-brain barrier and to increased sensitivity to drugs. Cell 77: 491-502; 1994.

106. Sikkema J, de Bont J, Poolman B. Mechanisms of membrane toxicity of hydrocarbons. Microbiological Reviews 59: 201$222 ; 1995$.

107. Ma D, Cook DN, Alberti M, et al. Genes acrA and $a c r B$ encode a stress-induced efflux system of Escherichia coli. Molecular Microbiology 16: 45-55; 1995.

108. Thanassi DG, Cheng LW, Nikaido H. Active efflux of bile salts by Escherichia coli. Journal of Bacteriology 179: 2512-2518; 1997.

109. van Helvoort A, Smith AJ, Sprong H, et al. MDR1 P-glycoprotein is a lipid translocase of broad specificity, while MDR3 Pglycoprotein specifically translocates phosphatidylcholine. Cell 87: 507-517; 1996.

110. Bosch I, Dunussi-Joannopoulos K, Wu R-L, et al. Phosphatidylcholine and phosphatidylethanolamine behave as substrates of the human MDR1 P-glycoprotein. Biochemistry 36: 5685-5694; 1997.

111. Alloing G, Granadel C, Morrison DA, Claverys J-P. Competence pheromone, oligopeptide permease, and induction of competence in Streptococcus pneumoniae. Molecular Microbiology 21: 471-478; 1996.

112. Perego M. A peptide export-import control circuit modulating bacterial development regulates protein phosphatases of the phosphorelay. Proceedings of the National Academy of Sciences (USA) 94: 8612-8617; 1997.

113. Höltje J-V. Growth of the stress-bearing and shape-maintaining murein sacculus of Escherichia coli. Microbiology and Molecular Biology Reviews 62: 181-203; 1998.

Hendrik W. van Veen earned his B.Sc., M.Sc. and Ph.D. degrees in biochemistry and microbiology summa cum laude at the Wageningen Agricultural University, the Netherlands. In 1996, his thesis work on solute transport in bacteria was awarded the Kluyver Award of the Netherlands Society for Microbiology. After a post-doctoral research fellowship at the University of Oxford, UK, he received a fellowship from the Netherlands Academy of Art and Sciences to pursue research on microbial multidrug resistance as a project leader at the Department of Microbiology, University of Groningen, the Netherlands. He was recently awarded the Young Investigators Award by the Federation of European Biochemical Societies, and the Max Gruber Award in Biochemistry by the Groningen Biomolecular Sciences and Biotechnology Institute. Monique Putman received her M.Sc.at Wageningen Agricultural University in 1993. She is now working towards a Ph.D. degree in microbiology at the University of Groningen, and studies the molecular properties of the secondary multidrug transporter LmrP of Lactococcus lactis. Wim van Klompenburg earned his Ph.D. degree at the University of Utrecht, the Netherlands, in 1997. As a postdoctoral fellow subsidized by the Dutch Cancer Society, he is now at the Department of Microbiology, University of Groningen, studying transport mechanisms of the human Multidrug Resistance-associated Protein. Rene Heijne is working as a technician at the Department of Microbiology, University of Groningen, on the human Multidrug Resistance-associated Protein, subsidized by the Dutch Cancer Society. He received his M.Sc. at the University of Groningen in 1993. Abelardo Margolles received his Ph.D. degree from Santiago de Compostela University, Spain, and completed his thesis work in Food Microbiology at the Consejo Superior de Investigaciones Cientificas (CSIC) in 1997. He is now a postdoctoral fellow (European Community Program-TMR fellowship) at the Department of Microbiology, University of Groningen, studying molecular properties of the lactococcal multidrug transporter LmrA. Wil N. Konings is professor in Microbiology at the Department of Microbiology, University of Groningen, and group leader of the Molecular Microbiology research group. He received his Ph.D. degree in biochemistry in 1969 at University of Groningen. His career has included visiting professorships at the Glynn Research Institute (Bodmin, UK), the National Institute of Health (Bethesda, USA), the Roche Institute of Molecular Biology (Nutley, USA), and the John Curtin School of Medical Research, Australian National University (Canberra, Australia). He is a member of the Netherlands Academy of Arts and Sciences. 